\title{
Improved Bragg Peak Integration for Neutron Crystallography by 3D Profile Fitting
}

Brendan Sullivan ${ }^{\mathrm{a}}$, Rick Archibald ${ }^{\mathrm{b}}$, Leighton Coates ${ }^{\mathrm{a}}$, Xiaoping Wang ${ }^{\mathrm{a}}$, Franz Gallmeier ${ }^{\mathrm{b}}$, Jack Carpenter $^{\mathrm{a}}$, Vickie Lynch ${ }^{\mathrm{a}}$, Paul Langan ${ }^{\mathrm{a}}$

\author{
${ }^{a}$ Neutron Sciences Directorate, Oak Ridge National Laboratory, Oak Ridge, TN \\ ${ }^{a}$ Computing and Computational Sciences Directorate, Oak Ridge National Laboratory, Oak Ridge, TN
}

Neutron crystallography presents a unique opportunity for crystallographers that is yet to reach its full potential because high backgrounds and weak scattering cross sections render the resulting datasets difficult to analyze. To address this limitation, we demonstrate the use of three dimensional profile fitting of Bragg peaks in reciprocal space. By using an Ikeda-Carpenter function to describe the timeof-flight coordinate and a bivariate Gaussian to fit the remaining two directions, we obtain excellent agreement between the measured and modeled peak profiles. In this integration scheme, the peak and background are separated by setting the background level such that the resulting time-of-flight profile is consistent with the characterized moderator emission. Weak peaks are fit by applying the profile of a nearest neighbor strong peak. Three complete protein datasets have been fit, integrated, and refined. These datasets show improved merge statistics; in particular $\mathrm{CC}_{1 / 2}$ shows increases up to three-fold in high resolution shells by using profile fitting. Refinements on initial models show decreases in $\mathrm{R}_{\text {free }}$ of $2-2.5 \%$, confirming that profile fitting leads to improved refinement. Consistent with lower $\mathrm{R}_{\text {free }}$ values, nuclear density maps show better agreement with known structural features from X-ray crystallography and enable the visualization of new structural details. These results demonstrate that profile fitting has to potential to greatly improve the capabilities neutron crystallography. 